Original Articles

\title{
Implications of Carotid Arteriomegaly in Patients with Aortic Aneurysm
}

\author{
Toshihiko Iwamoto ${ }^{1,2}$, Akihiro Kimura' ${ }^{1,2}$, Toshinori Nakai ${ }^{1,2}$, Kiyoshi Kanaya ${ }^{2}$, \\ and Shin Ishimaru ${ }^{3}$ \\ ${ }^{1}$ The Brain \& Blood Vessel Laboratory, \\ ${ }^{2}$ Department of Geriatric Medicine, \\ ${ }^{3}$ Department of Cardiovascular Surgery, Tokyo Medical University Hospital, Tokyo, Japan.
}

\begin{abstract}
The pathophysiology of aortic aneurysm is complex and it has remained unclear how frequently arteriomegaly, a diffuse dilatation of the artery, is associated with aneurysm. Therefore, ultrasonic study of the carotid artery was conducted to clarify this issue in a large number of subjects. Carotid ultrasonography was performed in 1,108 Japanese men aged 50 or older, and the results of $\mathbf{3 7 9}$ patients with arteriosclerotic aortic aneurysm (AA) were compared with those of 211 patients with peripheral arterial disease, 65 patients with aortic dissection, 232 hypertensive subjects, and 221 normotensive subjects. The carotid diameter was measured bilaterally at two points on the common carotid artery, and we defined carotid arteriomegaly as an arterial diameter in the 95th percentile or above that in the normotensive control group according to the relevant age subgroups. The incidence of carotid arteriomegaly in the AA group $(25.9 \%)$ was significantly higher than in the other groups $(p<0.01)$ even when adjusted for body height and blood pressure. In the arteriomegaly subgroup, hypertension and cigarette smoking was significantly more frequent than in the nondilated artery subgroup. This study demonstrates that one fourth of patients with aortic aneurysm have arteriomegaly as a generalized systemic abnormality in the arterial wall. J Atheroscler Thromb, 2004; 11: 348-353.
\end{abstract}

Key words: Arteriosclerosis, Ultrasonics, Hypertension, Cigarette smoking

\section{Introduction}

The etiology of aortic aneurysm has been studied from the viewpoint of arteriosclerosis, hemodynamics, genetics, and inflammation $(1,2)$, although the incidence of arteriosclerotic aortic aneurysm has currently increased among those conditions. Pathological studies show that atrophy of the media with laceration of the elastic lamellae is common in the aneurysmal lesion $(1,2)$. Many vas-

All funds for this study were obtained from the first author's department.

Address for correspondence: Toshihiko Iwamoto, Department of Geriatric Medicine, Tokyo Medical University Hospital, 6-7-1 Nishishinjuku, Shinjuku-ku, Tokyo 160-0023, Japan.

E-mail: i-wam@ma.kcom.ne.jp

Received May 14, 2004.

Accepted for publication July 21, 2004. cular risk factors and atheromatous lesions have been known to contribute to the development of arteriosclerotic aortic aneurysm (1-4). Several studies (5-9), although in a small sample size, have also shown diffuse dilated artery, namely arteriomegaly, or increased stiffness of the artery apart from the aneurysmal lesion, suggesting generalized vascular involvement in patients with arteriosclerotic aortic aneurysm. However, recent study (10) demonstrated no increased diameter if adjusted for blood pressure, suggesting the need to adjust for background factors influencing the diameter and analysis in a larger sample size. Indeed, the diameter has been known to be influenced by age, sex, stature, and the condition at examination (11-13). Therefore, $B^{\circ}$ ultrasonic study of the carotid artery was conducted to clarify the frequency of carotid arteriomegaly in a large number of patients adjusted for background factors. 


\section{Subjects and Methods}

\section{Subjects}

A total of 1,108 Japanese men aged 50 or older were examined consecutively with carotid ultrasonography at our laboratory after routine tests including chest x-ray, abdominal ultrasonography, and determination of ankle brachial pressure index (ABPI) for the screening of aortic aneurysm (AA), aortic dissection (AD), and peripheral arterial disease (PAD). When aortic aneurysm and dissection were suspected on chest $\mathrm{x}$-ray or abdominal ultrasonography, further studies of the chest and abdominal computerized tomography or angiography or both were performed. AA was diagnosed based on these studies, which showed localized dilatation of the aorta more than $40 \mathrm{~mm}$ in diameter above the level of the renal arteries (aorta more than $30 \mathrm{~mm}$ in diameter below the level of the renal arteries). AD was diagnosed when intima-medial flap was identified in the images. Marfan syndrome, syphilis, and PAD with AA were excluded from this study.

When ABPI was less than 0.9 , angiography to diagnose $P A D$, in which severe stenotic changes were visualized, was done as well as in patients with history of intermittent claudication. This study was performed in accordance with the Helsinki Declaration of 1975 as revised in 1983.

Of 444 patients with dilated aorta, 379 patients had AA (AA group) and 65 patients had AD (AD group). Within 1 month after examination, 141 AA patients received artificial grafts, and all resected specimens were pathologically identified to be atheromatous lesions. In the $A D$ group, the Stanford types A and B were present in 15 and 50 patients, respectively. Of 211 patients with PAD (PAD group), iliac, femoral, and multiple obstructions were seen in 108,88 , and 15 , respectively. Two control groups consisted of 232 hypertensive subjects (HT group) and 221 normotensive subjects (NT group), which were confirmed not to have $A A, P A D$, or $A D$.

\section{Carotid ultrasonography}

Using ultrasonographic equipment (LOGIQ500 $\alpha$ with 8.0 $\mathrm{MHz}$ or more, GE-Yokogawa Medical Systems, Tokyo, Japan), all patients were examined by a single sonographer. As shown in Fig. 1, posterior oblique view at the cardiac end-systole was used to measure the interadventitial diameter of the carotid artery bilaterally on the common carotid artery at two points $2 \mathrm{~cm}$ and 3 $\mathrm{cm}$ proximal to the bifurcation (points 1 and 2, respectively). The reproducibility of our technique for determining the diameters at points 1 and 2 was examined for 238 randomly selected samples. The coefficients of variation between 2 measurements taken after an interval of 1 month were $2.8 \%$ and $2.6 \%$, respectively.

Dilatation of the common carotid artery was defined as an arterial diameter in the 95th percentile or above that

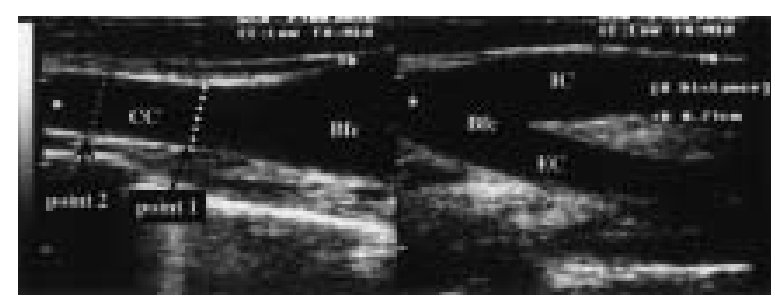

Fig. 1. Measurement of the carotid diameters at points 1 and 2.

The interadventitial diameter of the carotid artery was measured bilaterally on the common carotid artery (CC) at two points, $2 \mathrm{~cm}$ and $3 \mathrm{~cm}$ proximal to the bifurcation (Bfc). In the two images, IC indicates internal carotid artery, and EC indicates external carotid artery.

in the NT group according to the age subgroups as follows; subgroup aged 50-64 $(n=51)$, subgroup aged $65-$ $79(n=103)$, and subgroup aged 80 or older $(n=67)$. Based on the findings, carotid arteriomegaly was defined as having dilatation at four points, while partial dilated artery was defined as having dilatation at less than four points. The frequency of carotid arteriomegaly was compared not only among the groups, but also with adjustment for body height and systolic blood pressure.

Thickness of the intima-media complex (IMT) was also measured between the two points. Localized, thickened IMT of $2.1 \mathrm{~mm}$ or more in thickness (plaque) and stenotic lesions occupying more than $70 \%$ of the lumen diameter were assessed as carotid lesions (14).

\section{Cardiovascular risk factors}

Cases of hypertension were defined as those with a casual blood pressure $\geq 140 / 90 \mathrm{mmHg}$ or patients taking antihypertensive drugs. Diabetes mellitus was defined as fasting blood glucose concentration $\geq 126 \mathrm{mg} / \mathrm{dl}$ or patients receiving nutrition therapy and antidiabetic medication. Hyperlipidemia was defined as a fasting blood cholesterol concentration $\geq 220 \mathrm{mg} / \mathrm{dl}$ or cases receiving diet therapy and cholesterol-lowering medication. Cigarette smoking was defined as a smoking index of 200 or more (cigarettes/day $\times$ years smoking).

\section{Statistics}

Statistical analysis was performed by ANOVA, Fisher's protected least significant difference, and chi-square test with StatView-J 5.5 software (SAS Institution Inc., Cary, NC, USA). A $p$-value less than 0.05 was considered to indicate a statistically significant difference.

\section{Results}

\section{Patient characteristics}

There were significant differences in age, body weight, and blood pressure among the groups (Table 1). Regard- 
ing the $A A$ and $P A D$ groups, diabetes and cigarette smoking were more frequent in the PAD group than in the $A A$ group. The mean systolic blood pressure was higher in the PAD group than in the AA group, while the mean diastolic blood pressure was higher in the AA group than in the PAD group.

\section{Ultrasonographic findings}

Among the groups, the mean diameters of the carotid artery were largest at each point in the AA group $(p<$
0.05). (Table 2) The mean diameters in the PAD group were significantly larger than in the other three groups ( $p$ $<0.05$ ). The mean diameters in the AD and HT groups were also larger than in the NT group.

High frequency of carotid arteriomegaly was prominent in the AA group (25.9\%). In the other four groups, carotid arteriomegaly was more frequent in the PAD and AD groups. Adjusted for body height and systolic blood pressure, the frequency in each group showed a relation similar to the crude comparison, although no significant

Table 1. Background factors of each group.

\begin{tabular}{|c|c|c|c|c|c|}
\hline & AA group & PAD group & AD group & HT group & NT group \\
\hline No. of cases & 379 & 211 & 65 & 232 & 221 \\
\hline Mean age $\pm \mathrm{SD}, y$ & $72.2 \pm 7.0 * \dagger$ & $70.2 \pm 8.1^{\ddagger \ddagger \S}$ & $65.0 \pm 8.4$ & $72.0 \pm 9.5^{\dagger}$ & $73.5 \pm 9.8^{\dagger}$ \\
\hline Aged 65-79, $n(\%)$ & $267(70.4) \dagger$ & $127(60.2) \dagger$ & $24(36.9)$ & $95(40.9)$ & $103(46.6)$ \\
\hline Mean body height $\pm \mathrm{SD}, \mathrm{cm}$ & $163.2 \pm 6.3$ & $162.9 \pm 6.1$ & $163.7 \pm 8.1$ & $163.4 \pm 5.7$ & $163.0 \pm 5.9$ \\
\hline Mean body weight \pm SD, $\mathrm{kg}$ & $59.8 \pm 9.3 * \neq$ & $57.5 \pm 8.9 \ddagger \S$ & $66.0 \pm 13.0+\S \pi$ & $62.0 \pm 9.2$ & $60.4 \pm 10.1$ \\
\hline Mean SBP $\pm \mathrm{SD}, \mathrm{mmHg}$ & $136.8 \pm 16.0^{\S}$ & $141.9 \pm 16.2$ & $137.1 \pm 14.4 \neq \S$ & $143.3 \pm 15.9 \S$ & $130.2 \pm 12.2$ \\
\hline Mean $\mathrm{DBP} \pm \mathrm{SD}, \mathrm{mmHg}$ & $78.3 \pm 10.1 * \S$ & $76.4 \pm 9.1$ & $76.6 \pm 9.7$ & $78.2 \pm 9.4 * \S$ & $74.9 \pm 9.0$ \\
\hline Hypertension, $n(\%)$ & $269(71.0)$ & $145(68.7)$ & $58(89.2)$ & $232(100.0)$ & $0(0)$ \\
\hline Diabetes, $n(\%)$ & $53(14.0)^{\dagger}$ & $80(37.9) \neq \ddagger \S$ & $3(4.6)$ & $49(21.1)$ & $41(18.6)$ \\
\hline Hypercholesterolemia, $n(\%)$ & $118(31.1)^{\dagger}$ & $67(31.8)^{\dagger}$ & $13(20.0)$ & $91(39.2) \&$ & $54(24.4)$ \\
\hline Smoking, $n(\%)$ & $214(56.5)^{\dagger}$ & $171(81.0)+\neq \S \pi$ & $26(40.0)^{\S}$ & $98(42.2)$ & $55(24.9)$ \\
\hline
\end{tabular}

Abbreviations: AA: aortic aneurysm, PAD: peripheral arterial disease, AD: aortic dissection, HT: hypertension, NT: normotension, SBP: systolic blood pressure, DBP: diastolic blood pressure.

$* p<0.05$ vs PAD group, ${ }^{\dagger} p<0.05$ vs AD group, ${ }^{\ddagger} p<0.05$ vs HT group, ${ }^{\S} p<0.05$ vs NT group, " $p<0.05$ vs AA group

Table 2. Ultrasonographic findings of carotid arteries of each group.

\begin{tabular}{|c|c|c|c|c|c|}
\hline & AA group & PAD group & $A D$ group & HT group & NT group \\
\hline No. of cases & 379 & 211 & 65 & 232 & 221 \\
\hline \multicolumn{6}{|c|}{ Mean carotid diameter $\pm \mathrm{SD}, \mathrm{mm}$} \\
\hline Left: point 1 & $9.09 \pm 1.52 * * \ddagger \S$ & $8.83 \pm 1.13^{\ddagger \neq \S}$ & $8.47 \pm 1.34 \S$ & $8.18 \pm 1.04 \S$ & $7.81 \pm 0.98$ \\
\hline point 2 & $8.98 \pm 1.42 * * \neq \S$ & $8.65 \pm 1.15^{\ddagger \neq \S}$ & $8.24 \pm 1.41 \S$ & $8.00 \pm 1.04 \S$ & $7.64 \pm 1.05$ \\
\hline Right: point 1 & $9.29 \pm 1.54 * i \neq \S$ & $8.89 \pm 1.15 \neq \S$ & $8.56 \pm 1.31 \S$ & $8.24 \pm 1.13^{\S}$ & $7.97 \pm 0.93$ \\
\hline point 2 & $9.08 \pm 1.46 * * \neq \S$ & $8.70 \pm 1.34 \ddagger \S$ & $8.32 \pm 1.32 \S$ & $8.08 \pm 1.22 \S$ & $7.83 \pm 1.01$ \\
\hline Frequency of AM, $n(\%)$ & $98(25.9) * \hbar \ddagger \S$ & $23(10.9) \neq \S$ & $11(16.9) \neq \S$ & $8(3.5)$ & $3(1.4)$ \\
\hline Adjusted for body height & $122(32.2) * \neq \S$ & $41(19.4) \neq \S$ & $14(21.5) \neq \S$ & $5(2.2)$ & $7(3.2)$ \\
\hline Adjusted for SBP & $59(15.6) * £ \S$ & $17(8.1) \neq \S$ & $7(10.8) \neq \S$ & $5(2.2)$ & $6(2.7)$ \\
\hline \multicolumn{6}{|l|}{ Mean IMT $\pm \mathrm{SD}, \mathrm{mm}$} \\
\hline Left carotid & 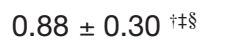 & $0.91 \pm 0.37 \ddagger \S \S$ & $0.72 \pm 0.21$ & $0.79 \pm 0.25 \S$ & $0.72 \pm 0.20$ \\
\hline Right carotid & $0.87 \pm 0.31 \ddagger \ddagger \S$ & $0.90 \pm 0.36 \neq \ddagger \S$ & $0.78 \pm 0.28$ & $0.76 \pm 0.20$ & $0.72 \pm 0.18$ \\
\hline Cases with plaques, $n(\%)$ & $198(52.2)$ & $141(66.8)^{\dagger}$ & $27(41.5)$ & $100(43.1)$ & $60(27.1)$ \\
\hline Cases with stenoses, $n(\%)$ & $33(8.7)+\pi$ & $40(19.0) \div \neq \S \pi$ & $1(1.5)$ & $14(6.0) \S$ & $4(1.8)$ \\
\hline
\end{tabular}

Abbreviations: AA: aortic aneurysm, PAD: peripheral arterial disease, AD: aortic dissection, HT: hypertension, NT: normotension, AM: arteriomegaly, SBP: systolic blood pressure, IMT: intima-media thickness.

$* p<0.05$ vs PAD group, ${ }^{\dagger} p<0.05$ vs AD group, ${ }^{\ddagger} p<0.05$ vs HT group, ${ }^{\S} p<0.05$ vs NT group, " $p<0.05$ vs AA group 
difference was seen between the $A A$ and $A D$ groups.

The mean thickness of the IMT in the AA and PAD groups was larger than in the AD, HT, and NT groups. Carotid lesions were more common in the PAD group, followed by the AA group.

\section{Characteristics of aneurysmal patients with arteriomegaly}

Carotid arteriomegaly was seen in $30.9 \%$ of thoracic AA, $23.2 \%$ of abdominal AA, and $32.0 \%$ of thoraco-abdominal AA. Compared with the non-dilated artery subgroup, a higher incidence of hypertension and smoking was seen in the arteriomegaly subgroup. (Table 3 ) Plaques and increased mean thickness of the IMT were frequent in the arteriomegaly subgroup.

\section{Discussion}

We studied Japanese men with the variables adjusted for body height and systolic blood pressure according to the age subgroups. Defining carotid arteriomegaly as above-mentioned, our study showed carotid arteriomegaly in one fourth of the AA group, which indicates the reason for the statistical increase in mean diameter of the carotid artery.

The carotid lumen often enlarges with increased steno- sis $(15,16)$, and our study showed increased frequency of plaques and more thickened IMT in the subgroups of arteriomegaly. However, plaques and stenoses were more common in PAD, and were mostly present in the internal carotid artery. Compared with the increase in diameter (1.2-1.3 mm), the differences in IMT between the arteriomegaly and non-dilated subgroups of the $A A$ group in one artery $(0.4-0.5 \mathrm{~mm})$ were small. Therefore, it is concluded that carotid arteriomegaly was not a compensatory change.

There are two types of arteriosclerosis of the large vessels; atherosclerosis and arteriosclerosis in a narrow sense, in which diffuse dilatation occurs with hardening of the vessel wall (17). According to this classification, arteriosclerotic aortic aneurysm is considered to be a form of dilated arteriosclerosis and usually, but not always, associated with atherosclerosis. Within aneurysmal lesions, histochemical study of the vessel wall metabolism showed decreased elastin volume with accelerated activity of elastase and matrix metalloproteinases $(1,2,18)$. Atrophy of the media and abnormality in elastin metabolism with loss of elasticity and fragility is suggested to be a major primary cause of arteriosclerotic aortic aneurysm, resulting in dilatation and rupture $(1,2)$.

It seemed that these aneurysmal changes may reflect those of carotid arteriomegaly in the AA group. His-

Table 3. Differences in background factors and ultrasonographic findings of carotid arteries among aneurysmal patients with or without arteriomegaly.

\begin{tabular}{|c|c|c|c|c|}
\hline & \multicolumn{2}{|c|}{ Dilated artery } & \multirow{2}{*}{ Non-dilated artery } & \multirow{2}{*}{$P$ value } \\
\hline & Arteriomegaly & Partially dilated & & \\
\hline No. of cases & 98 & 106 & 175 & \\
\hline Mean age $\pm \mathrm{SD}, y$ & $72.1 \pm 6.4$ & $73.5 \pm 7.2^{\dagger \ddagger}$ & $71.6 \pm 7.2$ & $p=0.0904$ \\
\hline \multicolumn{5}{|l|}{ Sites of aneurysm, \% } \\
\hline Thoracic, Abdominal aorta & $34.7,57.1$ & $31.1,61.3$ & $26.3,68.6$ & \\
\hline Thoraco-abdominal aorta & 8.2 & 7.6 & 5.1 & \\
\hline Mean body height $\pm \mathrm{SD}, \mathrm{cm}$ & $163.7 \pm 7.0$ & $161.9 \pm 5.6$ & $163.7 \pm 6.2$ & $p=0.0453$ \\
\hline Mean body weight $\pm \mathrm{SD}, \mathrm{kg}$ & $59.9 \pm 8.6$ & $58.5 \pm 9.8$ & $60.5 \pm 9.3$ & $p=0.2005$ \\
\hline Hypertension, $n(\%)$ & $85(86.7) * \dagger$ & $76(71.7)$ & $108(61.7)$ & \\
\hline Diabetes, $n(\%)$ & $11(11.2)$ & $15(14.2)$ & $27(15.4)$ & \\
\hline Hypercholesterolemia, $n(\%)$ & $26(26.5)$ & $40(37.7)$ & $51(29.1)$ & \\
\hline Smoking, $n(\%)$ & $62(63.3)^{\dagger}$ & $68(64.2)^{\dagger}$ & $74(42.3)$ & \\
\hline \multicolumn{5}{|l|}{ Mean IMT $\pm \mathrm{SD}, \mathrm{mm}$} \\
\hline Left carotid & $1.02 \pm 0.36 * \dagger$ & $0.90 \pm 0.28$ & $0.79 \pm 0.24$ & $p<0.0001$ \\
\hline Right carotid & $0.99 \pm 0.36 * \dagger$ & $0.89 \pm 0.28$ & $0.80 \pm 0.27$ & $p<0.0001$ \\
\hline Cases with plaques, $n(\%)$ & $73(74.5) * \dagger$ & 47 (44.3) & $78(44.6)$ & \\
\hline Cases with stenoses, $n(\%)$ & $4(4.1)$ & $14(13.2)$ & $15(8.6)$ & \\
\hline
\end{tabular}

Abbreviation: IMT: intima-media thickness.

$* p<0.05$ vs Partially dilated subgroup, ${ }^{\dagger} p<0.05$ vs Non-dilated subgroup, ${ }^{\ddagger} p<0.05$ vs Arteriomegaly subgroup 
tochemical analysis reported by Baxter et al. (19) showed that the ratio of elastin to collagen in aneurysmal patients was decreased in the non-aneurysmal vessel wall similar to that in the aneurysmal vessel wall. It indicated that both aortic aneurysm and carotid arteriomegaly is a manifestation of systemic abnormality of the media, but aneurysmal dilatation tends to occur at predisposing sites such as the abdominal aorta. The reasons for these predisposing sites are related to lower numbers of elastic lamellae, and to characteristic hemodynamics, in which pulse pressure increases at the abdominal aorta $(1,2)$.

Degradation of the elastic fibers in the media is usually associated with mechanical alteration in stiffness $(8-10)$. Sonesson et al. (10) showed increased stiffness of the carotid artery in patients with abdominal AA even without increased carotid diameter, indicating some discrepancy between morphological and biomechanical alterations.

In our study, hypertension and cigarette smoking was frequently associated with carotid arteriomegaly in the AA group. The findings suggested that these vascular risks could promote dilatation of the artery with underlying fragile media (1). Carotid arteriomegaly was frequent not only in the $A A$ subgroups, but in the $A D$ and $P A D$ groups. A common characteristic of these groups was also hypertensive patients with smoking habit, suggesting underlying pathophysiological conditions similar to AA in addition to atherosclerosis $(4,11-13)$.

\section{Study Limitations}

We set up the dilatation criteria on the basis of the data from normotensive subjects, although there is no universally accepted definition of carotid arteriomegaly. Furthermore, our study could not clarify the longitudinal changes of the lumen dilatation in relation to the degradation of the media.

\section{Conclusion}

Ultrasonic study showed carotid arteriomegaly in one fourth of patients with aortic aneurysm, which indicated that, in those patients, aortic aneurysm was a representative manifestation of a generalized systemic abnormality in the arterial wall, and that carotid arteriomegaly detected by ultrasonography can be an indicator of aortic aneurysm.

Acknowledgements: The authors are indebted to Assistant Prof. Raoul Breugelmans and Prof. J. Patrick Barron of the International Medical Communications Center of Tokyo Medical University for their review of this manuscript.

\section{References}

(1) MacSweeney STR, Powell JT, and Greenhalgh RM: Pathogenesis of abdominal aortic aneurysm. $\mathrm{Br} \mathrm{J}$ Surg, 81: 935-941, 1994

( 2 ) Patel MI, Hardman DTA, Fisher CM, and Appleberg $\mathrm{M}$ : Current views on the pathogenesis of abdominal aortic aneurysms. J Am Coll Surg, 181: 371382, 1995

(3) Cabellon S Jr, Moncrief CL, Pierre DR, and Cavanaugh DG: Incidence of abdominal aortic aneurysms in patients with atheromatous arterial disease. Am J Surg, 146: 575-576, 1983

(4) Auerbach $O$ and Garfinkel L: Athrosclerosis and aneurysm of aorta in relation to smoking habits and age. Chest, 78: 805-809, 1980

( 5 ) Tilson MD and Dang C: Generalized arteriomegaly: a possible predisposition to the formation of $a b$ dominal aortic aneurysms. Arch Surg, 116: 10301032, 1981

( 6 ) Ward AS: Aortic aneurysmal disease: a generalized dilating diathesis? Arch Surg, 127: 990-991, 1992

( 7 ) Mukherjee D, Mayberry JC, Inahara T, and Greig JD: The relationship of the abdominal aortic aneurysm to the tortuous internal carotid artery. Arch Surg, 124: 955-956, 1989

( 8 ) Makita S, Ohira A, Tachieda R, Itoh S, Moriai Y, Niinuma $\mathrm{H}$, Nakamura $\mathrm{M}$, and Hiramori K: Dilation and reduced distensibility of carotid artery in patients with abdominal aortic aneurysms. Am Heart J, 140: 297-302, 2000

( 9 ) Cheng KS, Tiwari A, and Morris R: The influence of peripheral vascular disease on the carotid and femoral wall mechanics in subjects with abdominal aortic aneurysm. J Vasc Surg, 37: 403-409, 2003

(10) Sonesson B, Hansen F, and Länne T: Abdominal aortic aneurysm: a general defect in the vasculature with focal manifestations in the abdominal aorta? J Vasc Surg 26: 247-254, 1997

(11) Bonithon-Kopp C, Touboul PJ, Berr C, Magne C, and Ducimetiere $\mathrm{P}$ : Factors of carotid arterial enlargement in a population aged 59 to 71 years:the EVA study. Stroke, 27: 654-660, 1996

(12) Jensen-Urstad K, Jensen-Urstad M, and Johansson $\mathrm{J}$ : Carotid artery diameter correlates with risk factors for cardiovascular disease in a population of 55-year-old subjects. Stroke, 30: 1572-1576, 1999

(13) Mannami T, Baba S, and Ogata J: Potential of carotid enlargement as a useful indicator affected by high blood pressure in a large general population of a Japanese city: the Suita study. Stroke, 31: 2958-2965, 2000

(14) Iwamoto T, Miyaji H, Shinozaki K, Koyama S, and Takasaki M: Changes in carotid atherosclerosis patterns detected by ultrasonography in Japanese 
elderly patients with aortic aneurysm. J Athroscler Thromb, 10: 13-18, 2003

(15) Steinke W, Els T, and Hennerici M: Compensatory carotid artery dilatation in early atherosclerosis. Circulation, 89: 2578-2581, 1994

(16) Polak JF, Kronmal RA, Tell GS, O'Leary DH, Savage PT, Gardine JM, Rutan GH, and Borhani NO: Compensatory increase in common carotid artery diameter. Stroke, 27: 2012-2015, 1996

(17) O’Rourke M: Mechanical principles in arterial dis- ease. Hypertension 26: 2-9, 1995

(18) Longo GM, Xiong W, Greiner TC, Zhao Y, Fiotti N, and Baxter BT: Matrix metalloproteinases 2 and 9 work in concert to produce aortic aneurysms. J Clin Invest, 110: 625-632, 2002

(19) Baxter BT, Davis VA, Minion DJ, Wang YP, Lynch TG, and McManas BM: Abdominal aortic aneurysms are associated with altered matrix proteins of the nonaneurysmal aortic segments. J Vasc Surg, 19: $797-803,1994$ 